\title{
A Multi-Modality Computer-Aided Framework Towards Postmortem Identification
}

\author{
Andreas Mang, Jan Müller ${ }^{1}$ and Thorsten M. Buzug \\ Department of Mathematics and Technology, RheinAhrCampus Remagen, Germany
}

A multi-modality framework for forensic soft-facial reconstruction based on computed tomography (CT) and magnetic resonance imaging (MRI) is presented. CT is used to acquire a virtual representation of a skull find and MRI templates provide the desired soft tissue information to produce a facial likeness of a deceased individual. Two main strategies are described. The first is based on a regularized non-linear warping technique using radial basis functions known as thin-plate splines in $3 \mathrm{D}$ space. The second is an automatic segmentation scheme based on active contours, which will provide a facial template that can be morphed onto the CT of the skull find. These approaches are presented in the framework of a forensic workplace.

Keywords: craniofacial reconstruction, forensics, nonlinear warping, segmentation, active contours, thin-plate splines, forensic workplace.

\section{Introduction}

Soft-facial reconstruction on anthropologic or forensic skull finds has been successfully performed for many decades. Traditionally, manual techniques employing clay or plasticine modelled onto the skull are used. The principal goals of this kind of work are twofold. On the one hand, anthropologists are interested in giving an impression of the appearance of famous persons in history - the Stoertebecker face reproduction is an example of such work or giving an idea of a Neanderthaler's visage as a artwork for a museum's exhibition. On the other hand, forensic anthropologists are working hand in hand with criminal departments to identify a person by means of a soft-facial reconstruction to be published. While the first goal offers some artistic freedom to the sculptor, the latter restricts him or her to the information that has been assembled within the criminal investigation. If the sculptor, for example, has been supplied with the skull only, without any indication of the structure and color of hair, it is recommended to leave the hair away and to show the reproduction of the face only. Nevertheless, one requirement is the same for both goals. Manual face reconstruction requires profound know-how in material preparation, a deep understanding of anatomy and anthropology as well as artistic talents and handicraft skills. It is therefore apparent that only a small community of anthropologic scientists is able to perform adequate face reconstructions. However, even very experienced anthropologic or forensic sculptors need weeks for this very complicated reconstructive work. For that reason computerassisted facial reconstruction is becoming more and more important to facilitate and speed up the process of finding the appropriate reproduction of an individual's facial appearance.

The key to a successful face reconstruction be it performed manually or computer-assisted - is the anatomical and anthropological knowledge of the human facial soft tissue thickness distribution. Research in the field of soft tissue depth measurement has been very active over the last three decades [1]. In order to approximate the shape of the face of a deceased person, it is mandatory to understand the correlation between bone and overlying skin. Obviously, the forensic sculptor never knows the exact relationship between any point of the skull find and the soft tissue thickness as the cranial bone does not carry this information directly. Nevertheless, it is actually possible to create post-mortem facial 
estimations, because the bone matrix is considered to support the soft tissue and it is possible to achieve average soft tissue depth at standardized points on the cranium (e.g. supraglabella, nasion, subspinale, supramentale, supraprogonion, ....).

Acquiring facial soft tissue depth is one of the most widely discussed issues in forensic sciences. And, a variety of different measurement strategies were carried out $[2,3,4]$. Most measurements are performed on cadavers. Within these studies, a needle is driven through the skin until the bone is encountered by the tip of the needle. This strategy must result in inaccuracies, because soft tissue depth information is produced as a sparse dataset only and, there are inevitable post-mortem alterations like dehydration or autolysis, i.e. the breakdown of living matter by the action of enzymes produced in the cells of the cadaver [1]. Another problem is the flesh deformation caused by the penetration of the needle. Therefore, current approaches focus on imaging modalities like ultrasound, computed tomography $(\mathrm{CT})$ or magnetic resonance imaging (MRI) to collect the tissue landmarks from living individuals (see e.g. [5]).

However, many computational methods for the estimation of the individual's post-mortem facial appearance still suffer from the sparse distribution of depth information across a subset of feature points. Even if the soft tissue depth is known at some salient anthropological points, actually, there is an infinity of them. The method proposed in this paper gives a solution to this fundamental problem of sparse soft tissue depth information. It is a multi-modality approach combining MR and CT imaging (see Fig. 1). Due to the fact that MRI especially is designed to show soft tissue, and the fat signal of the skin is very prominent in MR images,

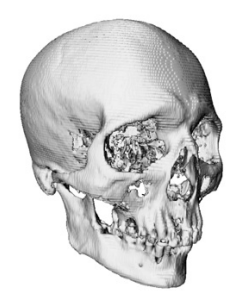

(a)

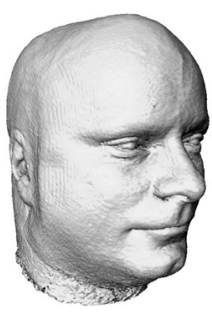

(b)
Fig. 1. We use (a) CT to acquire a virtual representation of the skull find and (b) MRI to measure facial templates that provide dense soft tissue distributions. the acquired data inherently carries the desired soft tissue depth information for the entire skull. Hence, we do no longer have to refer to charts providing lacunar depth information at about 20 or 30 standardized landmark positions.

In the application presented here, CT has been used to digitise the discovered skull as CT yields a virtual representation of the skull, which is free of any geometric distortion [6]. As a side remark, it should be noted that the acquired virtual representation of the skull can be employed as a basis for a so-called rapid prototyping. This technique allows the production of one-to-one plastic casts of the skull. These copies can be utilized for the traditional reconstruction using clay or plasticine, without putting the forensic or archaeological specimen at risk.

However, the aim of this paper is to give an overview on the state of the art of the transition from the traditional manual to the computerassisted reconstruction techniques, to present a registration-, as well as a segmentation-based face-reconstruction strategy and, finally, to introduce a prototype of a computer-based forensic workplace.

The paper is organized as follows. (i) the state of the art of computer-aided reconstruction methods is briefly reviewed in section 2 . (ii) the registration-based warping approach is presented in section 3. It is based on the non-linear warping technique using radial-basis functions known as thin-plate splines (TPS) extended to 3D space, i.e. a registration method first introduced by Bookstein in 1989 [7]. This landmark-based method, well known from registration studies in medicine, will be used to adjust the acquired MR soft tissue template to the CT cranial bone. To avoid errors induced by misplaced landmarks, a regularized version of this algorithm is used. (iii) The background to the segmentation-based approach is provided in section 4. It is a semi-automatic segmentation strategy extracting a facial template from MRI to be fit onto the CT data of the skull in a final morphing step. This approach is based on so-called deformable models or active contours, respectively, which are well known from image processing applications to locate object boundaries. They were first introduced by Kass, Witkin and Terzopoulos in 1987 [8, 9]. (iv) Finally, the prototype of a forensic workplace to facilitate the reconstruction procedure is presented in section 5 . 


\section{State of the Art in Computer-Aided Craniofacial Reconstruction}

This section will focus on principal developments in computer-aided craniofacial reconstructions and briefly review the state of the art. Basically, there are two fundamentally different methods for facial reconstruction, $(i)$ the anatomical and (ii) the tissue-depth methods $[10,11]$. The first approach is based on modelling the actual face muscles and the latter employing tissue-depth markers at standardized positions or landmarks, respectively, on the cranial bone. Anatomical approaches are considered to be extensively time-consuming since each individual muscle needs to be modelled separately and must be placed anatomically correct onto the skull [10]. However, most early computational methods presented in literature are indeed very similar to the manual approach based on dowels representing the soft tissue thickness. In contradiction, some recent approaches take advantage of the entire tissuedepth information.

In 1997 Archer [12] proposed to fit a generic hierarchical B-spline face model to a skull mesh according to predefined virtual landmark dowels representing the soft tissue thickness. In order to overcome drawbacks of applying splines to connect tissue-depth markers and in order to incorporate anatomy-based methods, so called non-uniform rational B-Splines (NURBS) are proposed in [13] to model the facial surface.

Andersson and Valfridsson [10] likewise employ landmark dowels defining the distance between skull and skin. All vertices of an arbitrarily chosen reference mesh are projected onto the CT representation of the cranial bone corresponding to the depth markers. Facial features can be chosen from a library and manipulated in a post-processing step. In order to make the model appear more lifelike, textures can be applied before rendering. One drawback of this approach is that the user needs to manipulate the mesh manually in order to insert or control facial features (e.g. one needs to identify all vertices defining the outline of the socket to introduce eyes to the mesh). Overall, the resulting faces appear slightly artificial.
Registration is very prominent in many medical image-processing applications to map one dataset onto another based on a certain correspondence between these different datasets. Thus, it is at hand to use registration-based approaches for craniofacial reconstruction as well. Moreover, Subsol and Quatrehomme [14] state that registration-based approaches are the most promising ones. Quatrehomme et al. [15], [14] employ single modality matching using a CT scan of the skull find and a CT scan of a reference skull. Additionally, a cast of the reference head soft tissue is incorporated to produce a facial approximation of the deceased individual. After registration the reference skull mesh is deformed parametrically according to so called crest lines onto the discovered skull. Applying the same mapping to the reference head the soft tissue cast is considered to result in the desired facial estimation.

Jones [16] suggests using cross-correlation to automatically identify feature points in the underlying datasets, i.e. the $\mathrm{CT}$ cranial bone and a CT reference head, respectively. Subsequently, this resulting correspondence is used to map the soft tissue of the reference head onto the skull find according to a so called distance-field warp function.

The approach presented by Bérar et al. [17] is similar to those presented by Quatrehomme et al. [15] and Jones [16] concerning the imaging modality used. However, a statistical shape model of skull and face is used instead of deformation-field calculation. Based on CT a database providing different skull surfaces associated with their corresponding skin surfaces is established. A series of tri-linear transformations is applied in order to register the different datas into a subject-shared reference system. In that way, a direct statistical model between face and skull is computed and subsequently used for the reconstruction of the deceased individual's face upon the CT cranial bone representation.

Tu et al. [18] use a set of CT scans to acquire tissue-depth information. Different reference CT scans from a database are morphed onto the subject's CT cranial bone representation. In order to obtain an average face and associated principal deformation modes according to each reference dataset, a principal component analysis of the aligned faces is carried out. Subsequently, the user has the opportunity to deform 
the average face corresponding to the contribution of the chosen deformation mode. Postprocessing allows altering features such as nose, eyes and lips and, in addition, texture mapping might be applied in a final step.

Similar to Tu et al. [18], Vandermeulen et al. [19] use multiple reference skin and skull surfaces. According to the method presented by Jones [16] a warping of a reference to a target skull is applied to a reference skin. For each CT in the reference database the facial surface and the skull surface need to be segmented and afterwards are transformed into signed Distance Transform (sDT) maps. Subsequently, these warps are applied to the target skull's sDT. Finally, the zero level set of their arithmetic average is defined as the reconstructed target skin surface.

Claes et al. [20] present an approach similar to the one proposed by Müller et al. [21]. A statistical facial model is calculated from different CT scans of living individuals in order to produce an unbiased facial template. Subsequently, this template is fitted onto the digital copy of the skull according to a set of corresponding landmarks. In particular, the method employs a minimal bending thin-plate-spine deformation (see section 3 ).

All the methods described above generally yield promising reconstruction results. One drawback that all these methods suffer from is that they take advantage of CT to acquire the necessary soft tissue information. It is quite hard to generate a satisfying reference database, as most cranial CT scans of living individuals are only taken in a limited field of view in order to reduce X-ray exposure (see Fig. 2.). Even with a low-dose CT scanning protocol, it is not wise - and in many countries simply not allowed - to acquire CT data from healthy volunteers. Therefore, in the methods proposed in the next sections, MRI is used instead of CT to form a soft tissue database.

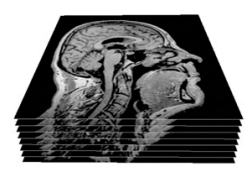

(a)

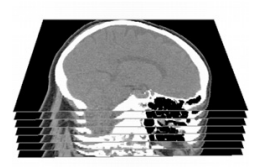

(b)
Fig. 2. Part of a typical stack of an MRI and of a CT dataset of the head.
Kähler et al. [11] present another registrationbased approach. It consists of an anatomybased virtual head model, incorporating skin and muscles to a scanned skull using statistical data on skull-tissue relationships. Based on Rhine's tissue-depth tables [2, 3] landmark dowels are set on the volume CT scan of the skull find and, using non-rigid registration, the reference head is deformed onto the virtual cranium. Moreover, underlying muscles are incorporated into the computed model and thus, this approach seems to be the first one integrating facial expression into computer-aided facial reconstruction. The resulting reconstruction is considerably nice. However, Kähler et al. state that, due to the limited number of landmarks, the computed deformation does not properly align the skin to the skull in all places. Therefore, additional landmark pairs are computed automatically by interpolation between existing ones.

Chen [22] presents an interface which might complement existing methods. In contrast to other approaches, this method does not change features of the face in order to achieve a positive identification. Starting from a central average face, the user has the possibility to choose from a set of related faces the one that resembles the desired face most closely. Each choice will arrive in a new set of related faces. Thus, the user is iteratively guided towards the desired facial estimation. This method takes advantage of faces produced by the FaceGen Software Development Kid package.

For a comprehensive historical overview of threedimensional reconstruction methods, see Tyrrell et al. [23] and the book of Clement and Marks [24].

\section{3D Soft Tissue Warping}

Due to dose considerations CT is used for digitization of the skull find only. In contrast to the methods reviewed in the section above, a facial soft tissue template is obtained from MRI. In that multi-modal way, inherently, the anatomical reconstruction method is carried out, because modelling of the actual face muscles is done by warping. 
This section deals with a non-linear registrationbased approach to produce a facial likeness of a deceased individual. Particularly, we use a 3D extended implementation of Bookstein's thinplate splines (TPS). It falls within the category of global basis-function methods. In general, it is an interpolation method for non-rigid registration of two sets of homologous anatomical landmark points in an irregular spacing, which need to be identified in both, the MRI and the CT datasets. Subsequently, the MRI dataset carrying the soft facial template is warped onto the CT scan of the cranial bone. As traditional methods require a precise knowledge of the anatomy of the face, the thickness of soft tissue at salient anthropological points and the relationship between various key features, such as eyes, nose, lips, chin, ears, in terms of proportion [1], using MRI as a second modality is at hand, because MRI already contains these features in an anatomically correct relationship.

\subsection{Mathematics in Brief}

This subsection provides a brief overview of the algebra of regularized 3-dimensional TPS; for a comprehensive coverage of 2-dimensional TPS we refer to Bookstein [7].

A volume is deformed by the interpolation of a so called correspondence function. The general problem can be stated as follows. A function $f$ must be found, which defines the continuous transformation $\mathbb{R}^{3} \rightarrow \mathbb{R}^{3}$ of all points $\mathbf{p}_{T}$ of the target volume (i.e. the CT cranial bone) onto their corresponding locations $\mathbf{p}_{S}$ in the source volume (i.e. the MRI of the reference head).

$$
\mathbf{f}\left(\mathbf{p}_{T}\right)=\mathbf{p}_{S}
$$

The labels target and source are related to the role of both volume data sets in the actual implementation. For each voxel in the target volume $T$ the method calculates the corresponding coordinates of the source data $S$ and copies the relevant gray value information found at these locations back to $T$. Therefore, any voxel of $T$ contains a gray value even if the volume is enlarged during transformation.

If we consider TPS in a physical manner, they can be compared with a thin metal plate being deformed by external forces. The more the plate is bent, the higher is the bending energy. If we permit small deviations only, in the absence of gravity, such a plate will bend in such a way that the associated physical bending energy $\mathcal{J}$ (eq. (2)) is at minimum [7]. In general, this behaviour can be described with one of Duchon's seminorms of appropriate dimension and order $[25,26]$. For the deformation of a volume dataset a seminorm of third dimension and second order is used [27].

$$
\begin{aligned}
\mathcal{J}(f) & =\iiint_{\mathbb{R}^{3}}\left(\frac{\partial^{2} f}{\partial x^{2}}\right)^{2}+\left(\frac{\partial^{2} f}{\partial y^{2}}\right)^{2} \\
& +\left(\frac{\partial^{2} f}{\partial z^{2}}\right)^{2}+2\left(\frac{\partial^{2} f}{\partial x \partial y}\right)^{2}+2\left(\frac{\partial^{2} f}{\partial x \partial z}\right)^{2} \\
& +2\left(\frac{\partial^{2} f}{\partial y \partial z}\right)^{2} d x d y d z
\end{aligned}
$$

Note that in eq. (2) $f$ denotes a scalar field. The criterion for the vector $\mathbf{f}$ can be taken simply as the sum of bending energies for each component $x, y$ and $z[26]$.

$$
\mathcal{J}(\mathbf{f})=\mathcal{J}\left(f_{x}\right)+\mathcal{J}\left(f_{y}\right)+\mathcal{J}\left(f_{z}\right),
$$

Thus, the correspondence function can be separated into three components, one for each dimension:

$$
\mathbf{f}\left(\mathbf{p}_{T, i}\right)=\left[\begin{array}{c}
f_{x}\left(\mathbf{p}_{T, i}\right) \\
f_{y}\left(\mathbf{p}_{T, i}\right) \\
f_{z}\left(\mathbf{p}_{T, i}\right)
\end{array}\right]=\mathbf{p}_{S, i} ; i \in\{1, \ldots, n\}
$$

where $n$ denotes the number of landmarks in the source and in the target volume, respectively.

As stated above, an interpolant $\mathbf{f}$ needs to be determined among all possible functions $(i)$ satisfying eq. (1) and (ii) minimizing the bending energy $\mathcal{J}$ from eq. (2). Such an interpolation function must be continuously defined and will map each landmark from the target volume $T$ exactly onto its corresponding landmark in the source volume $S$; for $\mathbb{R}^{3}$ it is given by

$$
f_{\bullet}(x, y, z)=\mathbf{a p}^{T}+\sum_{k=1}^{n} w_{k} \cup\left(\left\|\mathbf{P}_{k}-\mathbf{P}^{T}\right\|\right),
$$

where $\mathbf{a}=\left[a_{0 \bullet}, a_{1} \bullet a_{2 \bullet}, a_{3 \bullet}\right]$ and $\mathbf{p}=[1, x, y, z]$ $\mathbf{P}=[x, y, z]$ and $\mathbf{P}_{k}$ denotes the $x, y$ and $z$ coordinates of the target points. 
The functional of eq. (5) consists of two parts. The first part determines an affine transformation in 3D and the second part comprises the local elastic deformation. As a straightforward extension of Bookstein's warping formula [7], it minimizes eq. (2). The way the volume data is deformed is controlled by the still unknown parameters a and w. $U(\mathbf{r})$ is the so called radialbasis function (RBF). Since we are dealing with a 3D implementation, the RBF is as follows (for other dimensions than $\mathbb{R}^{3}$ we refer to Kybic [26]):

$$
U(\mathbf{r})=f(x, y, z)=\|\mathbf{r}\|=\sqrt{x^{2}+y^{2}+z^{2}}
$$

The RBF is the fundamental solution of the so called biharmonic equation $\Delta^{2} f=0$ [7]; i.e., $U(r)$ must satisfy

$$
\begin{gathered}
\Delta^{2} U(\mathbf{r})=\left(\frac{\partial^{4}}{\partial x^{4}}+\frac{\partial^{4}}{\partial y^{4}}+\frac{\partial^{4}}{\partial z^{4}}+2 \frac{\partial^{4}}{\partial x^{2} \partial y^{2}}\right. \\
\left.\left.+2 \frac{\partial^{4}}{\partial x^{2} \partial z^{2}}+2 \frac{\partial^{4}}{\partial y^{2} \partial z^{2}}\right) U(\mathbf{r}) \propto \delta x \delta y \delta z\right)
\end{gathered}
$$

where $\Delta^{2}$ denotes the two times iterated bilaplacian operator and $\delta(x, y, z)$ is the Dirac distribution.

Further investigations on eq. (5) indicate the need of boundary conditions to ensure that local deformations do not affect the whole area.

$$
\begin{aligned}
& \sum_{k=1}^{n} w_{k \bullet}=0, \quad \sum_{k=1}^{n} w_{k \bullet} x_{k}=0, \\
& \sum_{k=1}^{n} w_{k \bullet} y_{k}=0, \quad \sum_{k=1}^{n} w_{k \bullet} z_{k}=0 .
\end{aligned}
$$

Again, $n$ denotes the number of landmarks and $\mathbf{w}=\left[w_{1}, \ldots, w_{n}\right]$ is the set of elastic parameters of eq. (5). These conditions restrict the translation and rotation of the elastic part, as they ensure that terms with a more than linear increase - far away from the landmarks - will not be taken into account. The different coefficients $\mathbf{a}=\left[a_{0 \bullet}, a_{1 \bullet}, a_{2 \bullet}, a_{3} \bullet\right]$ of the affine and $\mathbf{w}=\left[w_{1}, \ldots, w_{n}\right]$ of the elastic part, respectively, from the analytic solution can be determined (5) by solving the following system of linear equations [27]:

$$
\begin{aligned}
& \mathbf{K} \mathbf{w}^{T}+\mathbf{P a}^{T}=\mathbf{v} \\
& \mathbf{P}^{T} \mathbf{w}^{T}=0
\end{aligned}
$$

where $\mathbf{K}$ is a matrix containing the values of the radial-basis function, $\mathbf{v}$ is a matrix containing the source locations and $\mathbf{P}$ is a matrix containing the target landmarks.

Due to the fact that the landmark placement is affected by errors, it is not favourable to force the graph of the interpolation function $f$ to pass through these erroneous points. Instead, small deviations of the graph of $f$ should be allowed. In order to minimize the influence of these errors, a regularized TPS version was implemented. We slightly need to change eq. (9) [27].

$$
\begin{aligned}
& (\mathbf{K}+\lambda \mathbf{I}) \mathbf{w}^{T}+\mathbf{P a}^{T}=\mathbf{v} \\
& \mathbf{P}^{T} \mathbf{w}^{T}=0
\end{aligned}
$$

I is an identity matrix of appropriate size and $\lambda$ denotes the regularization parameter.

\subsection{Results and Discussion on 3D Soft Tissue Warping}

We have presented a multi-modality approach to the reproduction of a facial appearance for post-mortem identification. It is based on a 3D non-linear warping technique, which allows deforming an MRI reference template onto the CT data of the cranial bone of a deceased individual. This method is able to provide a solution to the fundamental problem of sparse information about the depth of the soft tissue, as we deform an acquired reference MRI dataset, which contains the complete information related to the soft tissue to be matched onto the cranial bone.

In our implementation the $\mathrm{CT}$ scan of the skull represents the target volume $T$ and, accordingly, the MRI dataset providing the soft tissue template represents the source volume $S$. A tri-linear interpolation is used to suppress artefacts which are likely to occur using a nearestneighbour interpolation. Our current implementation in a MatLab ${ }^{\mathrm{TM}} 7$ environment takes 10 minutes to map a typical $256^{3}$ volume dataset containing 30 landmarks on a $2.2 \mathrm{GHz}$ standard PC. First results can be seen in Fig. 3.

As demonstrated in Fig. 3, results are encouraging. In comparison to the initial dataset, the forehead has strongly been deformed. Though some facial features, such as the nose, remain, the entire expression of the face has changed. 


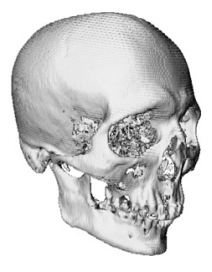

(a)

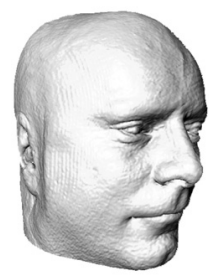

(c)

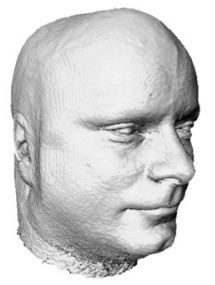

(b)

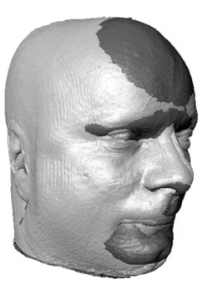

(d)
Fig. 3. Illustration of the warping of an arbitrarily chosen reference MRI (b) onto a CT cranial bone of an unknown deceased individual (a). In (c) one can see the resulting facial estimation and in $(\mathrm{d})$ the warped dataset is compared to the initial one.

Adding further individual spatial features like eyebrows, hair and further textures will yield nice reconstruction results. However, since the criminal investigation did not provide us with any further details about hair etc., the finishing make-up step is not carried out.

The main problem of the presented approach is the identification of corresponding landmarks in both datasets. As an option, a split view of sagittal, coronal and axial slices can be used, in order to set the corresponding anatomical landmarks in a three-dimensional spacing (see Fig. 4.). In some cases, this interaction scheme does not lead to adequate results. As an extension, the process of landmark setting is there-

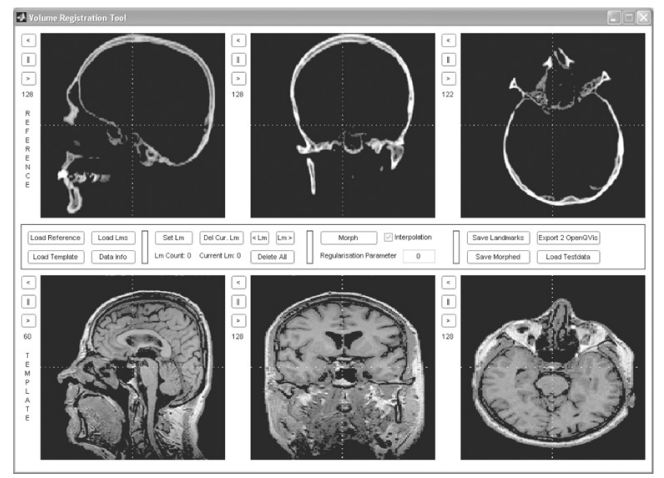

Fig. 4. Screenshot of the currently implemented Volume Registration Tool Software. fore integrated into a virtual three-dimensional visualization. Aditionally, it is recommended to set the homologous points in real world onto the cranial bone or rapid prototyping cast using an optical-tracking system. This leads to the concept of a forensic workplace that will be described in section 5 .

According to anthropological and anatomical analysis of the particular skeletal remains, an appropriate MR template needs to be chosen. This is an important step as TPS warping is not capable of all the transformations which are potentially needed, because only those transformations which preserve topology are possible. Therefore, an MRI soft tissue database must be built up containing different templates corresponding to ethnic groups, gender, age, weight, physiognomy etc., from which suitable initial templates can be chosen.

\section{Soft Tissue Measurements Based on Deformable Models}

This section addresses one of the most widely discussed issues in forensic sciences - the soft tissue measurements. In the following a segmentation strategy is presented to extract a facial template from a reference MRI dataset. This template provides the desired soft tissue-related information for the entire skull find and can be mapped onto the CT representation of the cranial bone in a final morphing step. A similar approach to the one presented below was introduced by Rifai et al. [28].

As illustrated in Fig. 5, the visualization of the MR fat signal of the skin yields two sharp boundaries. Detecting these edges will result in the desired soft tissue template. In a first step,

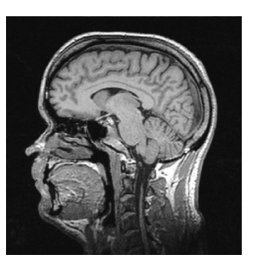

(a)

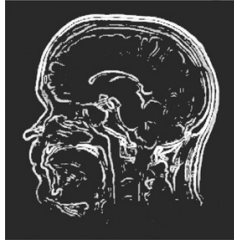

(b)
Fig. 5. This image illustrates the mid-sagittal section (a) and the Sobel filtered mid-sagittal section (b) through the head. As one can see in (b), two sharp boundaries limit the desired fatty signal of the skin. 
the object shape is mathematically described by so called active contours $[8,9]$. These deformable models are widely used in image processing applications to locate object boundaries. They move through the image domain by means of energy minimization towards salient features (usually object boundaries) under the influence of external and internal forces (see Fig. 6.). Additionally, some internal regularization constraints ensure the regularity of the curve and restrict its bending. Its physical analogue is a rubber band, which resists elongating and bending.

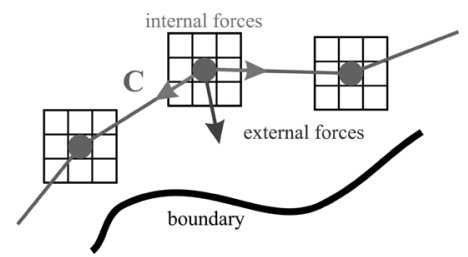

Fig. 6. Schematic snake movement through the image domain towards a boundary.

In the following, a brief overview is given of the deformable models used in forensic application. A detailed review of active contours can be found in [29].

\subsection{Mathematics in Brief}

Generally, an active contour model is an energyminimizing spline guided by internal constraint forces and pulled by image forces toward features such as boundaries [8]. The contour is initialized with an arbitrary initial shape and location within the image domain. Due to their intrinsic trend of energy minimization, they deform into the desired shape and move towards the desired location to align the object boundary. There are two different versions of active contours; $(i)$ the geometric or implicit and (ii) the parametric or explicit deformable models. In contrast to the parametric models, which are local methods based on an energy minimization procedure guided by external forces, geometric models can be regarded as zero level sets of a higher dimensional function, produced in an energy minimizing way [30]. These implicit models are based on the level-set method and on the theory of curve evolution, respectively. In the following, we will speak of active contours in the sense of parametric deformable models.

In $2 \mathrm{D}$ an active contour is a curve $\mathbf{C}(s)$ which can be described by

$$
\mathbf{C}(s)=\{\mathbf{x}(s)=(x(s), y(s)) \mid s \in[a, b]\},
$$

where $\mathbf{x} \in \mathbb{R}^{2}$ denotes the coordinate vector, which embeds the curve in $\mathbb{R}^{2}$ and $s \in[a, b]$ is the arc length of $\mathbf{C}$ parametrizing the contour. The complete energy function $\mathcal{E}$ guiding the deformation of $\mathbf{C}$ within the spatial image domain is as follows

$$
\mathcal{E}=\int_{b}^{a} \mathcal{S}(\mathbf{x}(s)) d s+\int_{b}^{a} \mathcal{P}(\mathbf{x}(s)) d s \stackrel{!}{=} \min
$$

$\mathcal{S}(\mathbf{x}(s))$ denotes the so called internal energy, which defines the physical properties of the contour. Particularly, it is composed of two terms

$$
\begin{gathered}
\mathcal{S}(\mathbf{x}(s))=\mathcal{E}_{e}(\mathbf{x}(s))+\mathcal{E}_{b}(\mathbf{x}(s)) \\
\mathcal{S}(\mathbf{x}(s))=\alpha(s)\left\|\frac{\partial \mathbf{x}(s)}{\partial s}\right\|^{2}+\beta(s)\left\|\frac{\partial \mathbf{x}^{2}(s)}{\partial s^{2}}\right\|^{2}
\end{gathered}
$$

where $\alpha(s)$ and $\beta(s)$ are non-negative weightingparameter functions determining elasticity and rigidity, respectively. Accordingly, $\gamma$ from (15) and (16) might as well be altered with respect to $s$. For simplicity $\alpha(s), \beta(s)$ and $\gamma(s)$ are commonly implemented as constant non-negative regularization parameters.

The first order term in (14) makes the contour behave like a membrane (i.e. it resists stretching), while the second term in (14) makes the contour act like a thin plate (i.e. it resists bending) [31]. Correspondingly, the first derivative in (14) defines the deformation along the tangential direction at $\mathbf{x}(s)$ and the second derivative defines the deformation of its curvature at that point. $\mathcal{E}_{e}$ is at minimum, if the first derivative disappears, i.e. $\mathbf{x}(s)=$ const. Thus, $\mathcal{E}_{e}$ tends to shrink $\mathbf{x}(s)$ towards a single location. The bending energy $\mathcal{E}_{b}$ represents the curvature and, therefore, it tends to be smoothen $\mathbf{C}$.

$\mathcal{P}(\mathbf{x}(s))$ from eq. (12) denotes the potential associated to the external forces along $\mathbf{C}$. It is computed directly from the image $I(x, y)$; if we consider a grey level image with continuous position variables $(x, y)$ typical potential energies are as follows [8] 


$$
\begin{gathered}
\mathcal{P}(x, y)=-\gamma|\nabla I(x, y)|^{2} \\
\mathcal{P}(x, y)=-\gamma\left|\nabla G_{\sigma}(x, y) * \nabla I(x, y)\right|^{2}
\end{gathered}
$$

where $\gamma$ is a non-negative weighting parameter, $\nabla$ is the gradient operator and $G_{\sigma}(x, y) *$ $\nabla I(x, y)$ denotes the convolution between the gradient of the image $I$ and a two-dimensional Gaussian function $G_{\sigma}(x, y)$ with standard deviation $\sigma$. The larger the standard deviation $\sigma$, the larger is the capture range of the external energy. Regardless of the choice of the exact external energy, the method for minimizing the $\mathcal{E}$ in $(12)$ is the same. Finding a curve $\mathbf{C}$ that minimizes the associated energy functional is a so called variational problem [29]. Each curve that balances the internal and external energies needs to satisfy the following Euler-Lagrange equation:

$$
\begin{aligned}
& \frac{\partial}{\partial s}\left(\alpha(s) \frac{\partial \mathbf{x}(s)}{\partial s}\right)-\ldots \\
& \ldots-\frac{\partial^{2}}{\partial s^{2}}\left(\beta(s) \frac{\partial^{2} \mathbf{x}(s)}{\partial s^{2}}\right)-\nabla E_{x}=0
\end{aligned}
$$

In order to find the solution of $(17) \mathbf{x}(s)$ is made dynamic and thus, must be treated as a function of time $t$. The partial derivative of $\mathbf{x}(s)$ with respect to $t$ needs to be set equal to the left-hand side of equation (17) [29]. This results in

$$
\begin{aligned}
& \eta \frac{\partial \mathbf{x}(s, t)}{\partial s}=\frac{\partial}{\partial s}\left(\alpha(s, t) \frac{\partial \mathbf{x}(s, t)}{\partial s}\right) \\
& -\frac{\partial^{2}}{\partial s^{2}}\left(\beta(s, t) \frac{\partial^{2} \mathbf{x}(s, t)}{\partial s^{2}}\right)-\nabla P(\mathbf{x}(s, t)) .
\end{aligned}
$$

The coefficient $\eta$ needs to be introduced to match the units of the left-hand side to the units on the right-hand side. As soon as the solution of $\mathbf{x}(s, t)$, which must be found numerically, converges, i.e. the contour reaches a steady state, all energies are at equilibrium. Accordingly, the left-hand side of (18) vanishes and, hence, the solution of equation (12) is found.

\subsection{Results and Discussion on Deformable Models in Forensic Applications}

The current implementation is based on a greedy algorithm. This method that computes the solution of (12), was first proposed by Williams et al. [32]. As mentioned above, the underlying problem is the segmentation of the head's soft tissue, as dense information about soft tissue depth is required in the forensic application.

The algorithm is organized as a two-step method. In the first segmentation step, the contour shrinks from the image boundary onto the outer boundary of the support of the image, which is essentially the skin surface of the head (see Fig. 7(b)). This first step is typically uncritical. However, as the skin thickness is needed, the inner boundary of the skin's fat signal is required as well. Here it is proposed to use the result of the first segmentation step as initial contour of the second step. To start further iteration towards the inner skin boundary, the high-pass filter signal of the outer skin edge is erased in the filtered image, i.e. the external energy at the corresponding image area is forced to be zero. In that way, the curve evolves to the inner boundary of the MR fat signal of the head. This procedure yields encouraging results at most locations. However, due to blurry edges, sparse edge information and high curvature next to the nasion and the chin, the results in these areas are unsatisfactory (see Fig. 7(b)) and need interaction.

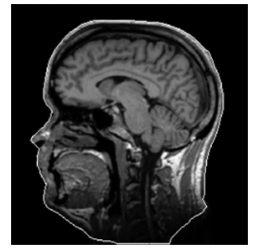

(a)

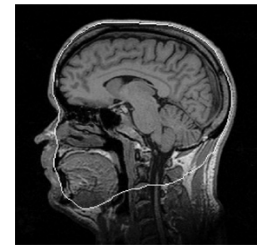

(b)
Fig. 7. First segmentation results of the template providing the desired information of the depth soft tissue. The active contour is coloured white.

A clear way to improve segmentation results is to use CT instead of MRI to obtain the desired tissue-depth information, since CT provides the desired template as well, without containing complex tissue structures. Nevertheless, we strongly recommend using MRI instead of $\mathrm{CT}$, as we do not believe that it will be possible in future to create a sufficiently large CT database of facial soft tissue templates.

Considering the advantages of geometric deformable models such as their intrinsic behaviour and their parameterization independence [33], the achieved results will benefit from a level 
set-based approach which is currently implemented. Moreover, a three-dimensional implementation of the presented method will positively affect segmentation results, as the deformable model is additionally influenced by edge information present in neighbouring slices of the volume dataset.

Once the MR template is extracted, it might be matched onto the CT representation of the cranial bone with the thin-plate spline method described in section 3. However, as an option the template may be morphed with a slightly modified algorithm utilized for the segmentation. The key idea is to equip a curve to be iterated with the active contour algorithm with one further attribute in addition to rigidity and elasticity, which is the soft tissue thickness. In that way, a curve can be morphed onto the CT data of the skull find that inherently carries the skin information.

As a side remark, it should be noted that this morphing step will face problems in the presence of dental artefacts in the $\mathrm{CT}$ reconstruction of the cranial bone. These CT-metal artefacts, which occur due to strong beam hardening, result in artificial stripes when a standard filtered backprojection is used for image reconstruction (see Fig. 8). However, since the presence of stripes caused by metallic cavity fillings in teeth is a diagnostic problem in CT as well, iterative reconstruction methods are currently implemented to reduce metal artefacts [34].

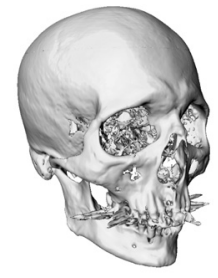

(a)

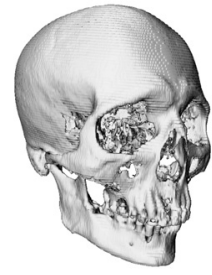

(b)
Fig. 8. Computed Tomography of a cranial bone (a) with dental artefacts and (b) without dental artefacts.

\section{Forensic Workplace}

As facial reconstruction requires a large amount of anatomical and anthropological knowledge, the main purpose of this part is to facilitate the process of creating an anatomically correct face reproduction. Besides the software modules described in the sections above, the presented prototype of a forensic workplace consists of a skull fixation unit, a PC with volume rendering extension card and an optical navigation system (Fig. 9.).

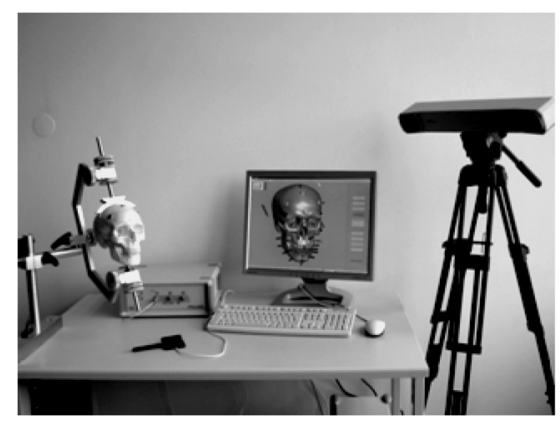

Fig. 9. The forensic workplace.

Due to the large and detailed datasets, the PC features a volume rendering card consisting of several highly specialized processors and a huge amount of memory to guarantee a steady workflow. In this case, it is a VolumePro 1000 card from TeraRecon, which is one of the leading volume rendering solutions at present. The clamp device for the cranial bone is inspired by a medical solution used for the fixation of the head during brain surgery. In order to simplify the interaction between forensic scientist and virtual data, the manipulation is done in real world using an optical navigation system. As the coordinates of the pointing device are updated several times per second, the opportunity of real-time interaction is provided.

The workflow of a reconstruction is as follows. (i) the cranial bone is fixed in the clamp and (ii) guided by the software, the user iteratively identifies predefined landmarks on the cranium using the pointing device; (iii) a proper MRI dataset, which already contains the corresponding landmarks, is chosen from the database and (iv) the warping is computed; $(v)$ textures might be applied to make the reconstruction results look more lifelike.

Thus, interaction and manipulation are becoming more intuitive to users with less artistic skills and less knowledge in anatomy and anthropology. Moreover, the system is able to provide instant feedback to the user. In order to preserve the specimen by any means, it is advisable to put a rapid-prototyping copy of the skull into the fixation device instead of the skull find itself. 
The segmentation-based method presented in section 4 will also be embedded into the forensic workplace. It will be combined with an originally B-spline-based reconstruction approach, that made use of the optical-tracking system to set virtual dowels representing the soft tissue depth at salient points on the CT representation of the cranial bone Fig. 10. According to Archer [12], B-splines are used to interpolate the soft tissue thickness between these dowels in order to produce a facial estimation.

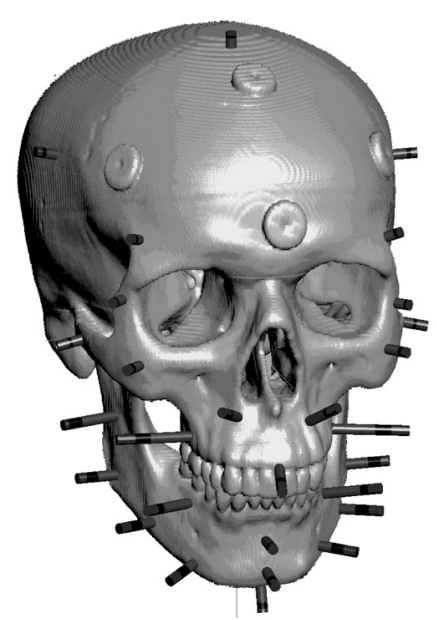

Fig. 10. Dowels representing the soft tissue thickness at standardized locations on the cranial bone.

As mentioned throughout the paper, the virtual dowels provide too sparse information on the soft tissue thickness. However, the methods described in the sections above, benefit from a combination with the dowels. The virtual dowels might be used to guide the shrinking process of the augmented contour, carrying the soft tissue related information. In addition, the dowels can be used to avoid rotation of the augmented contour, while morphing onto the skull. Furthermore, the dowels prevent the deformation of protruding parts of the face, such as the nose.

\section{Conclusion}

A framework has been presented towards the facial reconstruction for post-mortem identification. The methodology includes a registrationbased approach, which allows deforming a reference MRI soft tissue template onto the CT representation of the skull find. Furthermore, a method for segmenting the soft tissue depthrelated information from MRI was presented.

It has been demonstrated that the deformation of an MRI template based on non-linear warping leads to encouraging results and, moreover, it has been shown that active contours are capable of extracting a soft-facial template from MRI providing the required information on the depth of dense soft tissue for the entire CT representation of the cranial bone. Both, the registration as well as the segmentation-based approach, are integrated into a prototype of a forensic workplace that is inspired by the workflow of navigated surgery.

As MR and CT datasets are usually acquired from a lying individual, the facial shape, and therefore, the measured soft tissue depth, may differ from the one measured in upright position [1]. Studies will have to show to what extent these inaccuracies really affect reconstruction results.

Forensic facial reconstruction is a research area between art and science. Therefore, computeraided craniofacial reconstruction is actually a way to simplify the reconstruction procedure, but it will not be capable of replacing the intuition of the artist, which is highly demanded to achieve positive identifications.

\section{Acknowledgements}

The authors would like to thank Markus Pung and Stephan Theisen for their work in the field of image-guided navigation for forensic facial reconstruction. Additionally, we would like to thank Dirk Thomsen for the kind support in CT-image acquisition and Prof. Dr. Dr. Jürgen Ruhlmann for providing us with MR images.

\section{References}

[1] G. Quatrehomme AND G. SubSOL, Classical noncomputer-assisted craniofacial reconstruction. In Computer-Graphics Facial Reconstruction, chapter 2, page 1532. Academic Press, USA, April 2005.

[2] J.S. RHINE AND H.R. CAMPELL, Thickness of facial tissues in american blacks, Journal of Forensic Sciences, 25 (4) (1980), pp. 847-858. 
[3] J.S. RHINE AND C.E. MoORE, Facial Reproduction: Tables of Facial Tissue Thickness of American Caucasoids in Forensic Anthropology, University of New Mexico, Technical Report Maxwell Museum, Technical Series 1, Albuquerque, (1984).

[4] G.P. Helmer, S. Rohricht, C. Peterson ANd F. MoHR, Assessment of the reliability of facial reconstruction, Forensic Analysis of the Skull, Wiley-Liss, 17 (1993), pp. 229-246.

[5] V. PHILliPS AND N. SMUTS, Facial reconstruction: Utilization of computerized tomography to measure facial tissue thickness in a mixed racial population, Forensic Sci. Int., 83 (1996), pp. 51-59.

[6] T.M. BuZug, P. HERING AND R.P. HELMER, 3D tomography as a basis for anthropological and forensic facial reconstruction, K. Sigl and K. Prüfer Proceedings of the $1^{\text {st }}$ International Conference on Reconstruction of Soft-Facial Parts, Postdam, November, (2003), pp. 91-108.

[7] F. BooKsteIn, Principal warps: Thin plate splines and the decomposition of deformations, IEEE Transactions on Pattern Analysis and Machine Intelligence, 6 (11), June, (1989), pp. 567-585.

[8] M. KASS, A. WitKIN AND D. Terzopoulus, Snakes: Active contour models, International Journal of Computer Vision, 1 (4), (1987), pp. 321-331.

[9] D. Terzopolous, On matching deformable models to images, Topical Meeting on Machine Vision, Technical Digest Series (1987), pp. 160-163.

[10] B. ANDERSSON AND M. VALFRIDSSON, Digital 3D Facial Reconstruction Based on Computed Tomography, Linköpings Universitet, Sweden, February, (2005).

[11] K. KÄHLER, J. HABER AND H.P. SEIDEL, Reanimating the dead: Reconstruction of expressive faces from skull data, ACM TOG (SIGGRAPH Conference Proceedings), 22 July, (2003), pp. 554-561.

[12] K.M. ARCHER, Craniofacial Reconstruction Using Hierarchical B-Spline Interpolation, University of British Columbia, August, (1997).

[13] S.L. DAVY, T. GILBERT, D. SCHOFIELD AND M.P. EVISON, Forensic facial reconstruction using computer modelling software, JG. Clement and MK. Marks, Computer-Graphic Facial Reconstruction, 10 Academic Press, USA, April. (2005) pp. 183196.

[14] G. Subsol AND G. QuATREHOMme, Automatic 3D facial reconstruction by feature-based registration of a reference head, JG. Clement and MK. Marks, Computer-Graphic Facial Reconstruction, 5 Academic Press, USA, April, (2005), pp. 79-101.

[15] G. QUATREHOMME et al., A fully three-dimensional method for facial reconstruction based on deformable models, Journal of Forensic Sciences, 42 (1997), pp. 649-625.

[16] M.W. JONES, Facial reconstruction using volumetric data, Proceedings of the $6^{\text {th }}$ International Vision Modelling and Visualisation Conference, Stuttgart, November, (2001), pp. 136-142.
[17] M. BÉRAR, M. Desvignes, G. BAILly AND Y. PAYAN, 3D Statistical Facial Reconstruction, Proceedings of the $4^{\text {th }}$ International Symposium on Image and Signal Processing and Analysis (ISPA), Zagreb, September, (2005), pp. 365-370 .

[18] P. TU, RI. Hartley, W.E. LORENSEN, M. ALlyassin, R. GUPTA AND L. HEIER, Face reconstructions using flesh deformation modes ,JG. Clement and MK. Marks, Computer-Graphic Facial Reconstruction, 8 Academic Press, USA, April (2005), pp. 145-162.

[19] D. Vandermeulen, P. Claes, P. Suetens, S. De GREEF AND G. WILLEMS, Volumetric Deformable Face Models for Cranio-Facial Reconstruction, Proceedings of the $4^{\text {th }}$ International Symposium on Image and Signal Processing and Analysis (ISPA), Zagreb, September, (2005), pp. 353-358.

[20] P. Claes, D. Vandermeulen, P. Suetens, S. De GREEF AND G. WILLEMS, Statistically Deformable Face Models for Cranio-Facial Reconstruction, Proceedings of the $4^{\text {th }}$ International Symposium on Image and Signal Processing and Analysis (ISPA), Zagreb, September, (2005), pp. 347-352.

[21] J. Müller, A. MANG AND T.M. Buzug, A Template-Deformation Method for Facial Reproduction, Proceedings of the $4^{\text {th }}$ International Symposium on Image and Signal Processing and Analysis (ISPA), Zagreb, Semptember, (2005), pp. 359-364.

[22] T.-P.G. CHEN, 1,001,001 faces, a configural face navigation interface, University of British Columbia, June, (2003).

[23] A.J. Tyrrell, M.P. Evison, A.T. Chamberlain AND MA. GREEN, Forensic three-dimensional facial reconstruction: Historical review and contemporary developments, Journal Forensic Science, 42 (4) July, (1997), pp. 653-661.

[24] J.G. Clement AND M.K. MARKS, ComputerGraphic Facial Reconstruction, 10 Academic Press, USA, April, (2005).

[25] J. DUCHON, Interpolation des fonctions de deux variables suivant le principe de la flexion des plaques minces, RAIRO Analyse Numérique, 10 (1976), pp. 5-12.

[26] J. KYBIC, Elastic Image Registration using Parametric Deformation Models, Ecole Polytechnique Fédérale de Lausanne, Lausanne, Switzerland, (2001).

[27] K. ROHR, H.S. STIEHL, R. SPRENGEL, T.M. BUZUG, J. WEESE AND M.H. KUHN, Landmark-based elastic registration using approximating thin-plate splines, IEEE Transactions on Medical Imaging, 20 June, (2001), pp. 526-534.

[28] H. RIFAI, I. BLOCH, S. Hutchinson, J. WiART AND L. GARNERO, Segmentation of the skull in MRI volumes using deformable models and taking the partial volume effect into account, Medical Image Analysis, 4 (2000), pp. 219-233. 
[29] C. XU, D.L. PhAM AND J.L. PRINCE, Image Segmentation Using Deformable Models, JM. Fitzpatrick and M. Sonka, Handbook of Medical Imaging, 2 (3), SPIE press, May, (2000), pp. 129-174

[30] M. WEI, Y. ZHOU AND M. WAN, A fast snake model based on non-linear diffusion for medical image segmentation, Computerized Medical Imaging and Graphics, 28 (3), (2004), pp. 109-117.

[31] N. Paragios, O. Mellina-Gottardo and V. RAMESH, Gradient vector flow fast geodesic active contours, International Journal of Computer Vision, 1 (2001), pp. 67-73.

[32] D. Williams, D.J. SHAH AND M. SHAH, A fast algorithm for active contours and curvature estimation, CVGIP: Image Understanding, 55 (1), January, (1992), pp. 14-26.

[33] X. Han, C. Xu and J. Prince, A Topology Preserving Level Set Method for Geometric Deformable Models, IEEE Transaction on Pattern Analysis and Machine Intelligence, 25 (2003), pp. 755-768.

[34] M. OehleR, L. PfaffMAnN And T.M. BuZug, Artefact Suppression in Computed Tomography using Iterative Reconstruction Methods, Biomedizinische Technik 50, 1 Nürenberg, (2005), pp. 1130-1131.

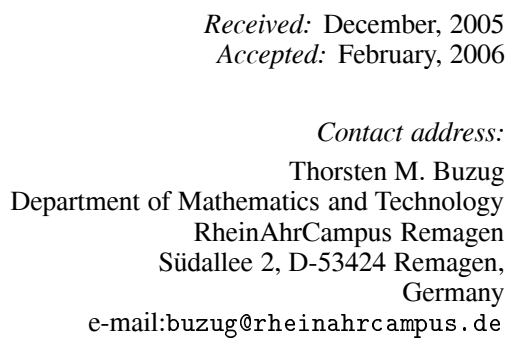

ANDREAS MANG is an undergraduate student at the Department of Mathematics and Technology at the RheinAhrCampus Remagen, Germany. Since 2002 he has been studying medical engineering at the RheinAhrCampus Remagen, University of Applied Sciences Koblenz. In 2003 he joined the Research Group of Prof. T. M. Buzug in the field of image processing for craniofacial reconstruction. He worked on the source reconstruction software package CURRY at Compumedics Germany $\mathrm{GmbH}$ during his practical term for about seven months in 2004/2005. He is member of the DGBMT (German Society of Biomedical Engineering) of VDE.

JAN MÜLLER is an undergraduate student at the Department of Mathematics and Technology at the RheinAhrCampus Remagen, Germany. Since 2002 he has been studying medical engineering at the RheinAhrCampus Remagen, University of Applied Sciences Koblenz. In 2003 he joined the Research Group of Prof. T.M. Buzug in the field of image processing for craniofacial reconstruction. Currently he is working on iterative reconstruction algorithms in the field of computed tomography. He is member of the DGBMT (German Society of Biomedical Engineering) of VDE.
THORSTEN M. BUZUG is a Professor at the Department of Mathematics and Technology, RheinAhrCampus Remagen, Remagen, Germany. He was born in Lübeck, Germany, in 1963. He received his DiplomPhysiker degree in 1989 and his Ph.D. in Applied Physics in 1993, both from the Christian-Albrechts-University of Kiel, Germany, where he worked in the field of signal processing applied to chaotic systems. From 1993 to 1994 he held a postdoctoral position at the German Federal Armed Forces Underwater Acoustics and Marine Geophysics Research Institute, where he worked on image acquisition and processing techniques for SONAR applications. In the end of 1994 he joined the Philips Research Laboratories Hamburg, Germany. He was the leader of the Philips Research Cluster Medical Image Processing and responsible for several projects in that field. In October 1998 he was appointed as professor of Physics and Medical Engineering at the RheinAhrCampus Remagen. From 2000 to 2004 he was head of the Academic Development Committee of the RheinAhrCampus Remagen. Since 2005 he is head of the Joint Council of Campus Departments and director of the Institute of X-Ray Optics (IXO) at RheinAhrCampus Remagen. He has published numerous journal articles, conference papers and books. He is member of Scientific Board of the Journal "Befund: Krebs" and of the Advisory Committee "Stiftung Sparkasse Zukunft Kreis Ahrweiler". He is member of the German Physical Society (DPG), German Society of Biomedical Engineering (DGBMT), the German Society of Nondestructive Testing (DGfZP) and the IEEE. 